\title{
GhanaToll: Barrier-Free RFID Toll Collection and Management System for Ghana
}

\author{
B. Kommey \\ Kwame Nkrumah University of \\ Science and Technology \\ KNUST-Kumasi \\ Ghana
}

\author{
C. Acquaye \\ Kwame Nkrumah University of \\ Science and Technology \\ KNUST-Kumasi \\ Ghana
}

\author{
T. A. Abeka \\ Kwame Nkrumah University of \\ Science and Technology \\ KNUST-Kumasi \\ Ghana
}

\begin{abstract}
Ghana like most developing countries has most of the toll booths across the country managed manually which more often than not is beset with many problems - the lack of transparency and accountability being main examples. Many at times personnel entrusted to run such systems mismanage the funds. In some cases, the daily sales are not properly accounted for and the infrastructure is not properly maintained. Considering various electronic tollbooths systems that have been deployed in many parts of the world and the innumerable advantages they bring to such countries; it is safe to say that this project will address the challenges faced in developing countries in relation to toll collection. This paper thus proposes the design of an electronic tollbooth collection and management system suitable for the local needs in developing countries particularly Ghana. The proposed system leverages the unique identification on Radio Frequency Identification (RFID) tags and uses it to identify a particular user's account that has been registered with the system. This unique identity read by the RFID reader is relayed to the microcontroller unit responsible for processing the data and transmitting it wirelessly to an online server in charge of identifying the account the unique ID is associated with and additionally handling the deductions from the specified user's account. Feedback from this process is sent back to the microcontroller unit so the user receives a response about the status of the transaction. By this, accountability is achieved causing a domino effect of reduced traffic congestion, reduced fuel consumption, and carbon monoxide emissions and as well as the reduction in the travel time.
\end{abstract}

\section{Keywords}

Electronic Toll Collection (ETC), Toll Gate, Radio Frequency Identification (RFID), Drivers Vehicle and Licensing Authority (DVLA), Toll Credits, Global System for Mobile Communications (GSM).

\section{INTRODUCTION}

Toll Booths were introduced to serve as a means of raising money to facilitate the construction of new roads and the maintenance of existing roads in Ghana. Jones A.K and Hoare R.R proposed this idea of toll and tax collection serving as one of the sources of funds for government and maintenance of roads in 1986. Toll personnel who sit in booths at the tollgates do the collection of tolls. Upon arriving at the gate, the drivers pay their tolls, which is dependent on the class of vehicles being driven. Receipts are issued after completion of payments. This system has a major flaw in that there is no effective way to ensure that the tolls collected correspond with the number of cars that use the gate at a particular time
[1]. As such, there is a possibility of some of the monies going unaccounted for. Currently in Ghana, there are quite a number of tollbooths available with at least one in a region; however, the Accra-Tema tollbooth is the only booth that has been made computerized in some way [2]. Looking at the different types of vehicles that are used in Ghana, a classification can be made to put them into three different classes. An interview was conducted and it was discovered that for saloon cars and other private cars, the toll charge is 50 pesewas per tollgate; however, that of $4 \times 4$ private cars and other vehicles within this range are charged GHC1.0. This same charge is given to the commercial vehicles popularly referred to in Ghana as 'trotro'. For buses, the charge given is $\mathrm{GHC1.5}$, while tipper trucks and other big articulator trucks pay an amount of GHC2.0. Trailers are charged GHC2.5 [3]. Considering the Accra-Tema motorway that has twelve (12) tollbooths with toll attendants, some assumptions can be made to estimate the number of tolls collected daily. Assuming 20,000 vehicles use the motorway in a day and assuming that every vehicle is charged at least GHC1.0 per trip, a revenue value of GHC20,000 can be realized in a day, GHC140,000 a week and a total estimated value of GHC560,000 in a month [4]. Deducting the cost of labor and other factors reduces the estimated amount hence the need for a system that properly accounts for these tolls collected as well as offering a cheaper alternative.

\section{RELATED WORKS}

The idea of automatic toll collection is not new. There have been attempts and some implementations by some countries and agencies. This section reviews some of the related work and examines their strengths and shortfalls.

S. Nandhini and P. Premkumar, 2007 proposed an automatic tollgate using advanced RFID and GSM technology [5].

This system employs the use of RFID and GSM in the collection of tolls. Capacitive sensors are used to detect the

size of the vehicle. IR sensors are used to detect the presence of a vehicle at the toll plaza and a Toll Tax Unit gate model is used to control the opening and closing of tollgates as a vehicle enters or exits. Information on the vehicle is stored in the memory of the microcontroller using the tag number. The tag is read by the RFID reader and based on the tag number the appropriate toll amount is deducted. Information on the transaction is sent to the phone of the owner using a GSM modem. The flaw of the proposed system is that the use of IR technology makes the system susceptible to failure. In addition, the use of capacitive sensors to determine vehicle size may result in the classification of a small but heavily packed vehicle as same as a bigger but lighter car. The two cars would then be required to pay same toll. Owners of 
vehicles would have to bear the cost of acquiring the RFID tag and finally, the implementation of the system would result in major changes in the architecture of existing toll roads.

W. Lee et al, 2018 implemented an electronic toll collection method based on vehicle positioning system [6]. This system employs the use of an On Board Component, Global Positioning System, an enforcement system and a backend system to ensure the collection of tolls. The mechanism operates based on the interaction between the On-Board Component and backend through GPRS. The flaw of the proposed system is that the VPS is a new technology; it is currently undergoing development and field trials worldwide and as such, there is no business operation instance in the world. It is more difficult in matching the process between the debit and enforcement information, so the VPS system needs more post-processing jobs in order to reduce the mismatch failure. In addition, the personal privacy problem is still there, just like Dedicated Short Range Communication based ETC. The implementation of this method proved to be much lengthier and very expensive than expected.

Ramkrishna Vadali et al, 2019 proposed a secured electronic toll collection using RFID and mobile application [7]. The RFID tag was deployed by the toll authority, by embedding a unique identification number (UIN) and the customer's details into the tag. The deployed active RFID tag is attached to the windshield of the vehicle. Whenever the vehicle passes through the tollbooth, RFID reader read the tag and data and send same to the server for verification. The server checks the tag details and depending upon the type of the vehicle, the toll amount deducted from the user's account. The notification about the toll amount deduction is then sent to the customer via SMS and email as well. An android software application was developed and used to recharge the customer's account. With the system, the flaws are that the low frequency results in lower maximum data rate, although it is fast enough to allow multiple transmissions to increase reliability. Passive RFID tags are relatively less adept than active RFID tags, while active RFID tags are expensive. The speed of the vehicle can bottleneck the system. If at a particular tollbooth user loses his network coverage, he may get SMS notification late.

A. Swathi and B. Masthan, 2018 described an RFID Based Automatic Toll Collection System using GSM Technology [8]. For this system, a $125 \mathrm{KHz}$ RFID peruser constantly scanning RFID tags was employed in recognizing the detached tag utilized by the client. When the tag connected to the vehicle arrives within the scope of the peruser, it advises the microcontroller and serially speaks with the tag. The tag is then identified after which the LCD will show 2 methods of activity; the exchange mode and revive mode. When the driver selects the exchange mode, with sufficient funds available, the vehicle stipulated charge is deducted. The revive mode allows the user choose between three buttons representing the amount he wishes to revive his account with. For this system, the maximum revive value is 250 . In both methods, the user receives an SMS with the help of the GSM modem. A DC Motor with an associated driver will open the entryway and the vehicle will go through the gate. The major flaw with this system is that in the event where the users doesn't have enough toll credit to pass through successfully, he will have to pause to select the revive mode which gives him access to press a button to get the necessary funds he needs to use the gate. This wait time coupled with the time it takes for the microcontroller to communicate with the driver to open the gate makes the system more time consuming as the traditional method of tolling.

R. Suganya et al, 2018 implemented an automated toll plaza system using RFID and GSM [9]. This system of tolling was completely dependent on using RFID to identify different vehicles. A unique tag is given out when a user registers with the system and it holds information about the user which is stored in a database and linked to a bank account.

At the tollgate, an RFID reader acting as an interrogator is positioned in a way to collect the information of the vehicle and its user with the help of the antennas placed together with the tags. These antennas generate signals that transmit the encoded data on the tag. The data is cross referenced with the data stored in the database after which the tax is deducted from the associated bank account. The deducted amount is displayed on an LCD screen at the gate after which stepper motors connected to the gate is activated to allow the vehicle access. An SMS is sent confirming the status of the transaction to the user. In the case where a vehicle is not registered with the system or tag id is not unique, the vehicle owner had to resort to traditional method of tolling where the attendants gives a ticket which he pays to allow access. This system of tolling also presented an additional feature that allows the detection of stolen vehicles. This is made possible when the owner of the stolen vehicle enters the details of the said vehicle on the website. There were security issues in accessing bank account details linked with a vehicle.

S. S. Malaj and S. S. Kamate, 2017 implemented an RFID and GSM Based Toll Tax System [10]. In this system unlike other RFID based systems, the new user can register himself and verify using a registered tag. When a driver drives to the gate, he is prompted to scan the tag. The reader indicates with a buzzer the particular card has been read. In an event the reader is unable to read tag, a camera captures the vehicle. Next the data that has been read is matched with the data existing in the card and stored in the code memory then it displays a message as "Authorized" in the first row of the LCD and "Wineyard welcomes" in the second row of the LCD after which another message displayed as "Gate Opens" is shown along with the DC motor rotating in Clockwise direction to open the gate.

The major issue apart from the time it takes the user to go through the above-mentioned process, is the fact that power is wasted. This is because when there is no vehicle at the gate, the LCD screen continuously displays 'Place card' till a vehicle drives up to the gate Also, after the user has paid the tax and the gate is opened, the system gives a delay which could be abused by other vehicles to pass through the gate without paying.

\section{MATERIALS AND METHODS}

\subsection{GhanaToll System Architecture}

As shown in figure 1 and 2, the proposed GhanaToll electronic toll collection and management system consists of an RFID reader, a microcontroller unit with a GSM shield and an online web server disseminating information to both system administrators and end-users. Each vehicle using the tollgate has been equipped with a unique RFID tag positioned on the windshield of the car. An RFID reader, connected to the microcontroller unit, obtains the RFID tag ID and then relays the read ID with the help of the GSM/GPRS shield wirelessly to the online database server. The whole system process depends on four major operations as shown in figure 3. 
These operations are:

- Vehicle Detection, where sensors placed at the base station detect the presence of a vehicle at the toll point or gate

- Vehicle Identification, where the vehicle in question is placed into a class based on a set of predefined categories,

- Automatic Toll Collection, where the appropriate toll charge is collected and

- Enforcement operation, which handles cases where the road user does not have enough funds in his prepaid account or in events where unregistered users utilize the ETC point or gate. The enforcement operation is activated with the aid of a camera after an interrupt is triggered when the reader is unable to read any Tag ID on the vehicle that has used the toll point. In this case, the camera takes an image of the vehicle and the driver. This image is then processed and used to identify the vehicle class in order to calculate the charge. Relevant information is forwarded to the Driver and Vehicle Licensing Authority (DVLA) so that the charge would be collected from the driver whenever he goes to renew his driver's license or roadworthy certificate.

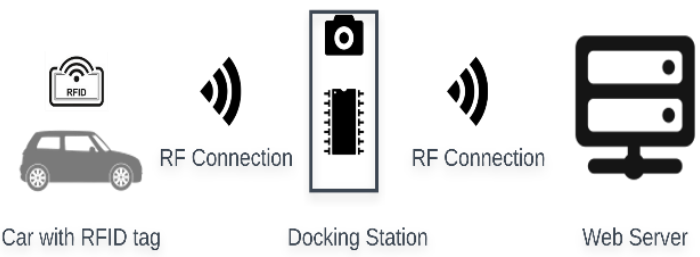

Figure 1. GhanaToll System Architecture -I

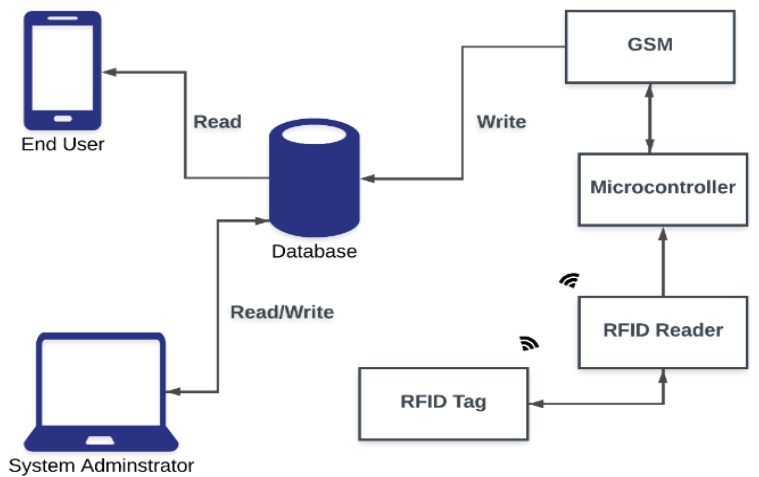

Figure 2. GhanaToll Collection System Architecture II

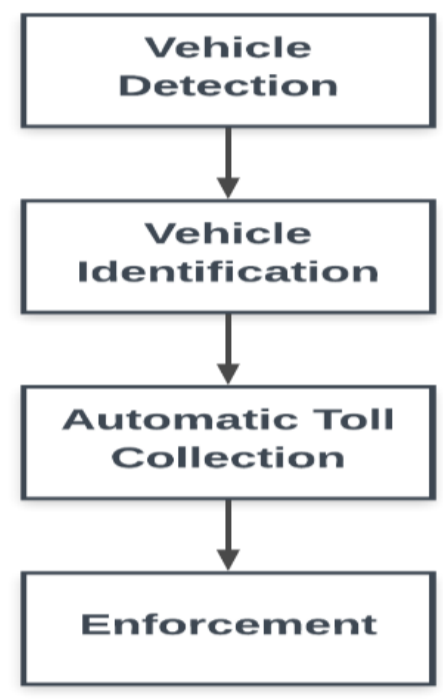

Figure 3. GhanaToll System Data Processing

\subsection{GhanaToll System WorkFlow}

In this paper various designs, based on their cost-effectiveness and accuracy were considered. As transmission plays a vital role in ensuring the efficient operation of the system, it is essential to consider technologies that help achieve the goal of the system while considering the aforementioned considerations. The GhanaToll system employs the Global System for Mobile Communications (GSM) protocol for communication between the microcontroller units and the online web server because it provides reliability as compared to using other wireless communication protocols and for the fact that Wifi signals are not always available in Ghana. The vehicle identification in this system relies heavily on the system's ability to relay effectively, the read ID of the vehicle, therefore SIM900A GSM module comes in handy. In addition to its functionality, the SIM900A is able to send SMS messages to confirm transactions to toll gate users and also connect to the internet and send data to the online database for backend transactions to happen.

Other techniques employed by the GhanaToll system includes:

-Radio Frequency Identification that is used for communication between the tag on the vehicle and the reader because RFID technology does not rely on line of sight for communication.

-Time-division multiple access (TDMA), which suits the system requirements since there are a number of vehicles accessing a shared medium but not at the same time. As such, the system allows all these tags to share the same channel frequency by allocating each tag its own time slot (depending on the time the vehicle reaches the gate) to send its signal.

Also, the fact that the reader communicates with a particular tag at a time, makes using RFID more convenient.

Figure 4 depicts the GhanaToll collection system flow chart 


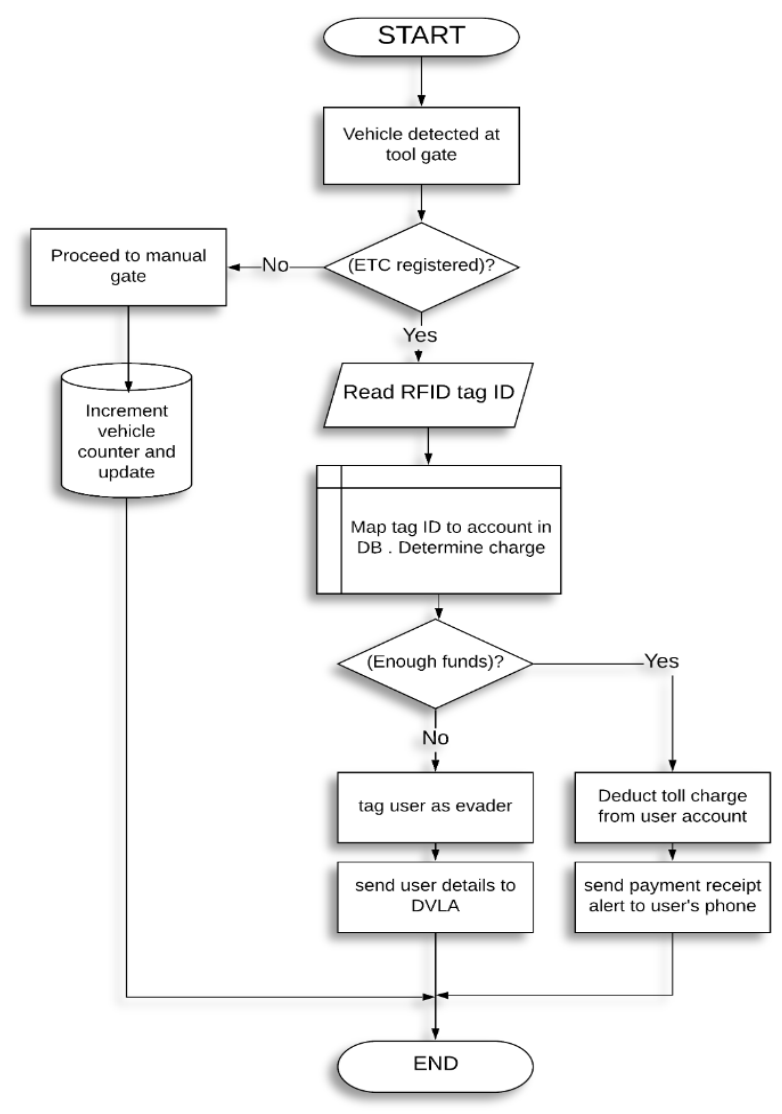

Figure 4. GhanaToll System Flow Chart

\subsection{GhanaToll System Hardware}

As shown in figure 5, the GhanaToll system hardware components are classified into the following main blocks: Input, Microcontroller Processing, Output, and Communication blocks.

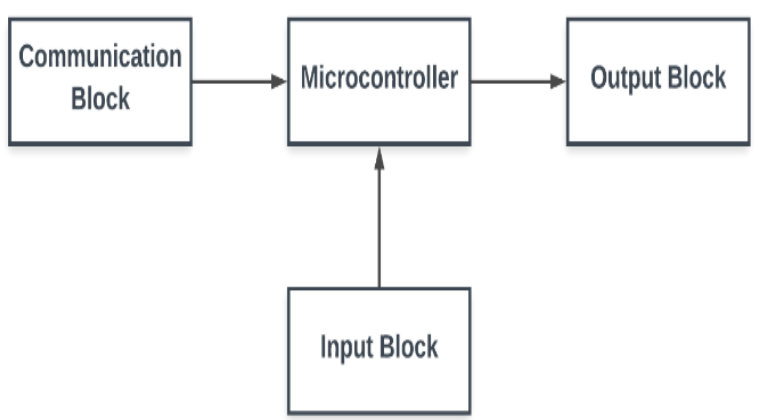

Figure 5. GhanaToll Hardware Block Diagram

The Input Block includes the reader that reads the information encoded on the tag attached to the vehicle. Additionally, it collects the camera events that are triggered only when unregistered users use the ETC gate. The unique ID read by the reader is then transmitted to the processing block via the input pins of the microcontroller unit.

The Microcontroller Processing Block is where the data received is processed in some way in order to produce a certain effect. This effect is sent to the Output Block where an indicator gives feedback as to how data is moved from the RFID Reader to the microcontroller unit and finally transmitted to an online database server for the transactions to be performed. Transaction is done via the Communication Block that houses the GSM shield that connects the microcontroller-processing block via the serial port. The input and output transactions are as depicted in figure 6.

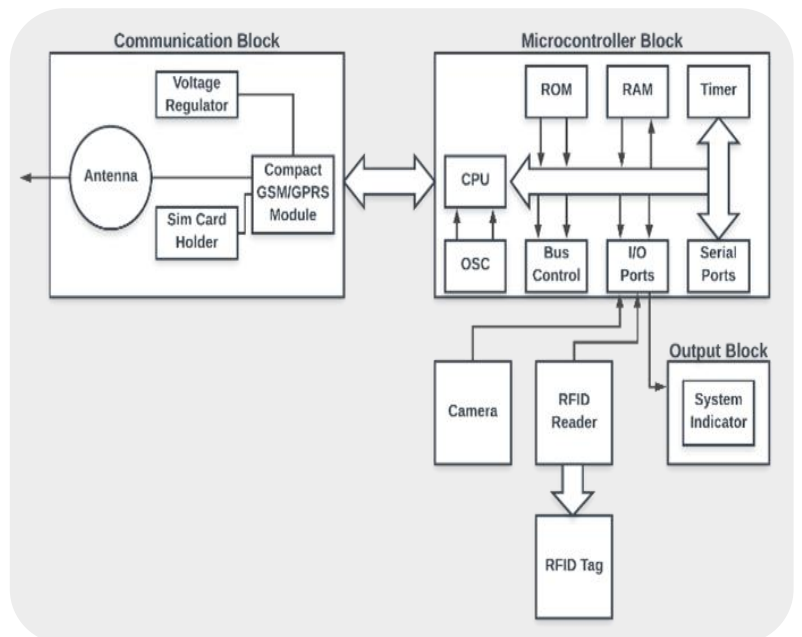

Figure 6. GhanaToll Functional Block Diagram

\subsection{GhanaToll System Software}

The GhanaToll system software application in its current form operates on different platforms. It includes a mobile application that runs on the Android OS or an iOS platform and a web-based application hosted on a browser for the administrators to monitor all tolling activities. This software application provides services not only for the toll collection agencies but also for the drivers utilizing the application.

These services include:

- Platform for drivers to scout different toll subscriptions available to them so they can make a choice based on their preference ranging from their choice of pricing and expiry dates posted by agencies selling toll subscriptions.

- Platform for interaction between toll collection agencies and drivers so any issues could be resolved or addressed.

- A feature that allows drivers to view their history of tolls paid in the past and their location on a map.

The users for this application have been grouped under two main categories: Toll Agencies and Toll Users, who in this case are the drivers using these toll points or gates.

Toll Agencies: This category of users are the ones who will be in charge of managing the whole toll collection process. This is to say that they will be responsible for the provision of the tags to be used in the system. A tag encrypted with a unique code, identifies a particular vehicle that is associated with it and an account that could be managed from the mobile software application by the registered driver. These Toll Agencies are also in charge of posting various toll subscriptions on the mobile software application so that registered drivers can purchase toll credits that could be automatically deducted from their accounts as and when they use a toll point or gate. They are also in charge of ensuring that estimated toll amounts for a particular period correlates with the actual calculated amount at the end of the period. Payment or Toll agents are trained to assist with any issues that may pop up from the end user's side. Figure 7 and 8 illustrate use case diagrams of an end user and a payment agent (administrator). 


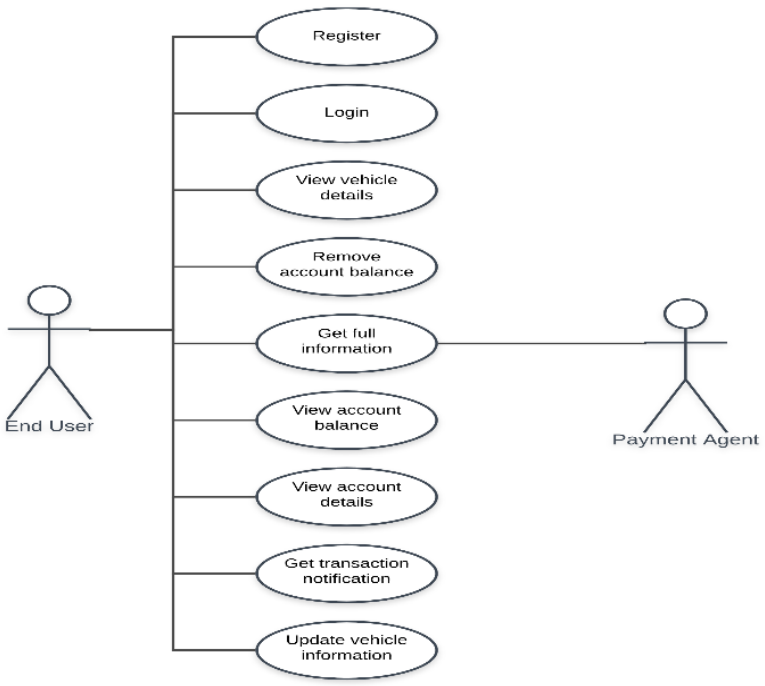

Figure 7. GhanaToll Application End User Use Case

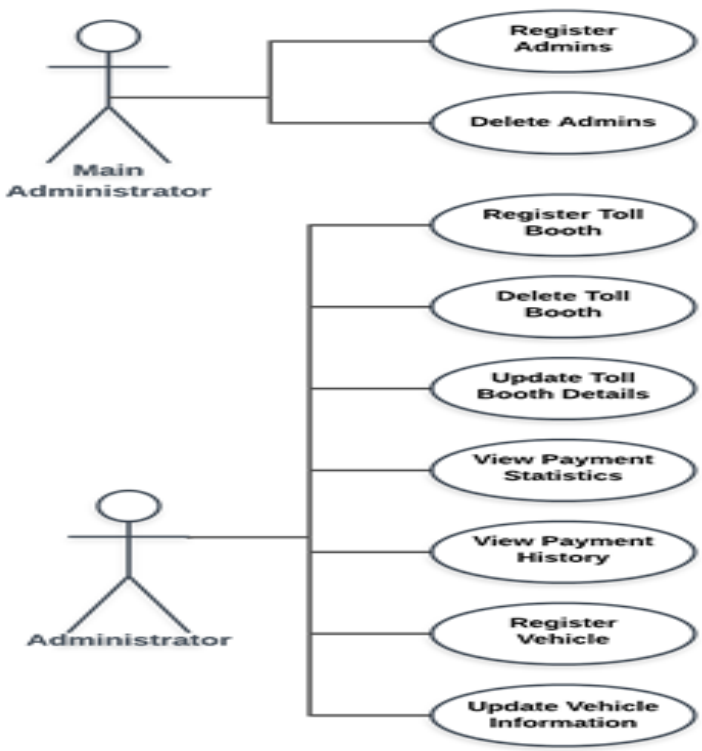

Figure 8. GhanaToll Application Admin Use Case

Drivers (Registered customers): These users also benefit from using the application; in that, they have access to any information they will require about a particular toll subscription posted by an agency. They can even go as far as interacting directly with a representative to ask about issues that may not be clear in the information posted. They then proceed to book a toll subscription-based on the information they have gathered. They also get the opportunity of obtaining an e-receipt that provides information about each transaction they make as they use the tollbooth. Additionally, they can view past toll information and view their toll credit balance. They will also be able to receive notifications when a toll transaction is successful. In the GhanaToll software application, PostgreSQL was used for database management. In addition, Flask was used to handle all other backend operations especially the RESTFUL APIs. As with any system, which comprises some hardware communicating with software, a strong backend needs to support the communication on the sides in question. The backend generally includes a web server that hosts the application and communicates with our software application that was hosted on Heroku.

The User Interface for the administrator was designed with tabs that are revealed after passed authentication. The Dashboard as shown in figure 9 provides a general overview of the system including the total amount of tolls collected within a day, month or year depending on the option selected by the user compared to the total estimated toll amount and the vehicles recorded at the toll gate. The registered tollgates tab presents information on all toll gates that have been registered under the GhanaToll system. For the purpose of the project, the Accra-Tema motorway was the only registered tollbooth. The Evader list shows all the users who passed with insufficient balance to pay their tolls. Users who redeem their tolls, by the design of the system will have their names removed from this list. The user subscriptions tab shows the administrator all the toll plans he has presented to the users and the number of the people who have activated that particular offer. The registered customers' tab gives the administrator information about all the customers who have registered with the system including information about the vehicles they have registered among others. The registered vehicles tab gives information about all the vehicles registered with the system. The help center tab presents a forum where administrators can address issues posted by customers.

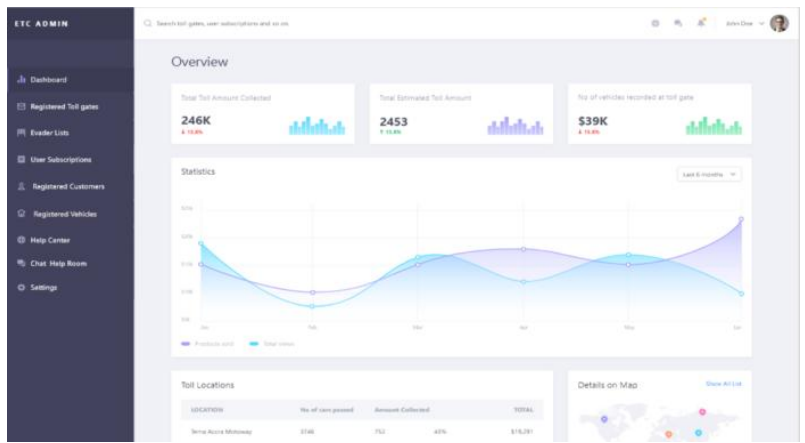

Figure 9. GhanaToll User Interface Dashboard

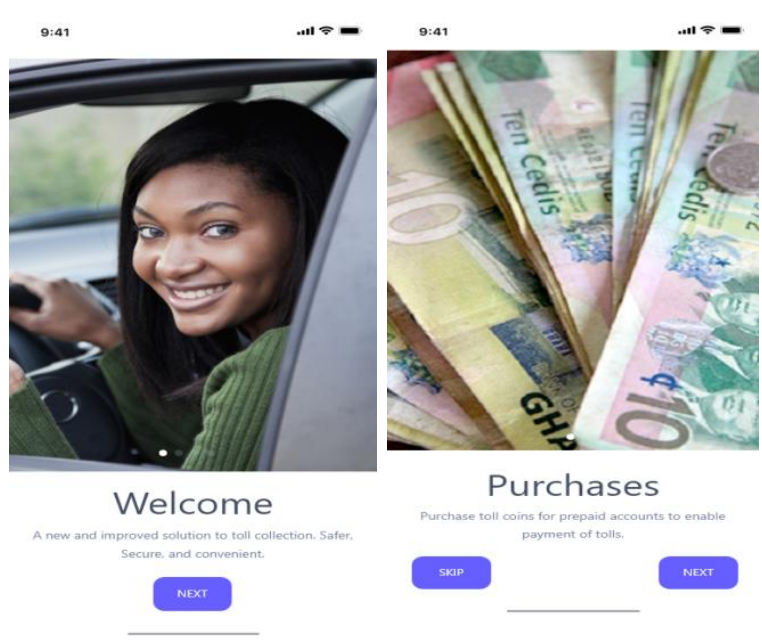

Figure 10. GhanaToll Introductory Slider

In figure, 10 above are screenshots that give the new user some information about the application he or she will be using so they would not be completely overwhelmed with the software environments. The GhanaToll mobile software application provides three main functions including the purchases, transactions and support feature. It is essential to highlight this when the registered driver first uses the 
application. The purchase slider communicates that the user can make a purchase of toll credit using any payment platform he considers necessary. The transaction slider communicates that the user can view information concerning his payments made in the past. Lastly, the support section highlights the fact that the various Toll Agencies would be available to assist the users in whatever way they could in relation to using the GhanaToll system. The software application starts with these screens after which signup and a sign-in screen will be provided so as to first allow the users to $\log$ in or for new users to sign up to use the system. After the details entered by the user has passed the authentication, the user is directed to the home page that contains two menu icons to allow the user access to notifications and to open the side navigation menu.

The next screen displayed in figure 11 is the side navigation which provides menu options like the history section, where the user can view his past transactions if any to allow him or her monitor his or her transactions. Information such as the location of the tollgate is provided in the form of a map, in addition, the name of the tollgate as known to the system is provided. The amount paid is also presented as well as the date and time the transaction took place.

The second menu option dubbed 'payments' presents the user with an interface where he can view his remaining toll balance for a particular subscription made. This is to help users monitor their toll credits so that they can make new subscriptions as and when necessary. Additionally, the payment options screen will also present the user with different subscriptions that have been posted by the various Toll Agencies so that based on the price and duration presented they can make a decision as to which subscription to choose. Selecting a particular subscription redirects the user to another page where they can select the payment option they prefer and make payments for the toll credit.

The next menu option 'Account Settings' will provide the user with the interface displayed in figure 11 .

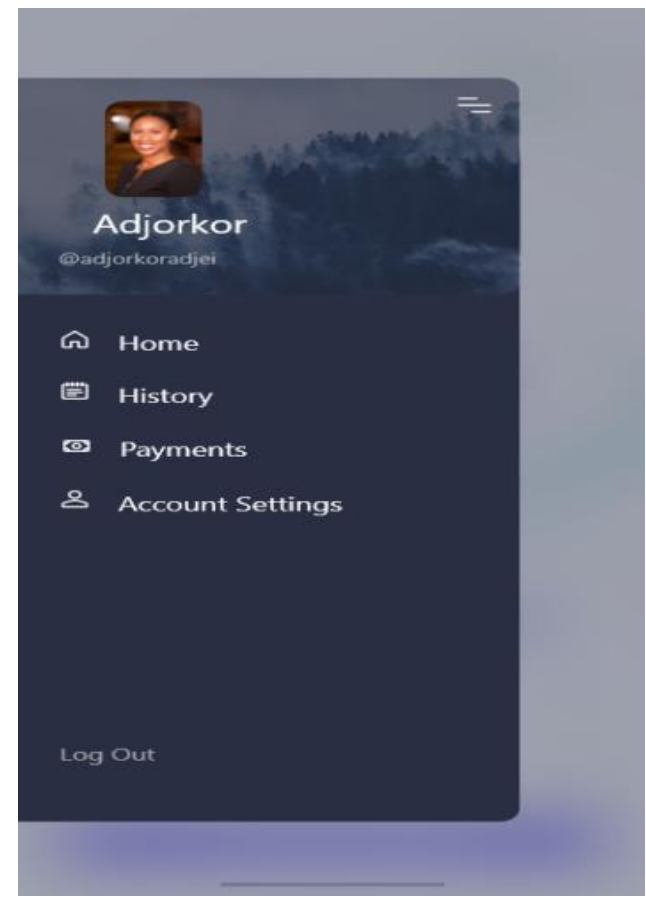

Figure 11. GhanaToll User Interface Side Navigation
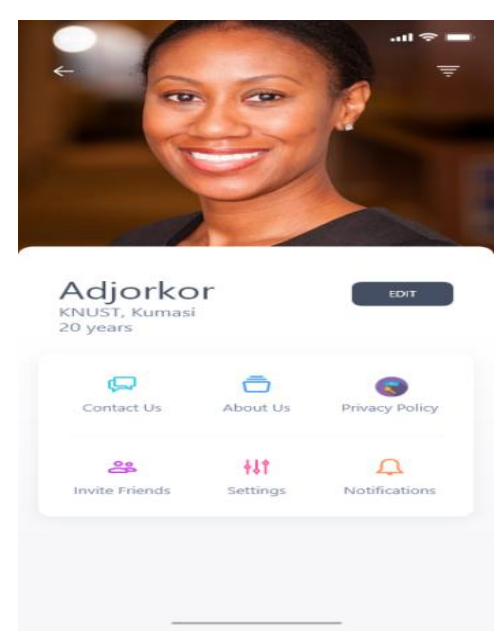

Figure 12. GhanaToll User Interface Profile Screen

The Account Setting tab opens a screen as shown figure 12 to allow the user to view different menu icons that directs the user to.

- Invite a friend to use the GhanaToll system or mobile software application.

- Interact with a representative from the Toll Agencies to assist them in case of issues.

- Notifications menu where the user can click and be directed to a page where they can view all notifications sent to them. Notifications may come in different forms in that user may receive notifications when they make a purchase or when a transaction has been made.

- General settings allow the user to make changes to the physical appearance of the application such as changing the size of the text among other functionalities that will make him have a more custom environment space to work with. The remaining menu options provide information about the terms, conditions that must be heeded to in addition to the privacy policy of the GhanaToll system.

\subsection{GhanaToll Embedded Software}

Apart from the user interface software described above, there is an embedded system software, which controls the hardware, and all activities of the hardware components. The source code contains logic that facilitates the harvesting of signals generated because of the sensor detecting the presence of a vehicle at the tollgates. The following subheadings discuss the relevant instructions used in the prototype of the embedded system.

\section{RFID Read}

This event is initiated by enabling SPI communication. The code that controls the behavior of the RFID reader like any component that connects to a microcontroller starts with first including the library that contains the functions that help us use the component after which the connected components pins are defined.

Additionally, the different card IDs are obtained and used in the prototype to enable the registration of Tags used for the system. The code below checks for the presence of any card and gets the UUID, if a vehicle is present at the tollgate. 
def get_vehicle_uuid():

\# Create an object of the class MFRC522

MIFAREReader $=$ MFRC522. MFRC522()

\# Scan for available tags

(status, TagType) $=$ MIFAREReader.MFRC522_Request (MIFAREReader.PICC_REQIDL)

\# If a vehicle is found at the gate

if status $==$ MIFAREReader. MI OK:

print ("Card Detected")

\# Get the UUID of the vehicle tag

(status, uid) $=$ MIFAREReader.MFRC522 Anticoll()

\# Select the scanned tag

MIFAREReader.MFRC522 SelectTaa(uid

\section{GSM/ GPRS Initialization and Transmission}

The code that initializes the GSM/GPRS board comes with some predefined AT commands. By sending out these AT commands, the board is able to send and receive data.

After initialization, the URL of the database where data would be sent was specified. Before that, the private keys for the handshake protocol are sent out, after which the specific URL with the port is also sent. A phone number is specified for use in the prototype.

After the transaction is completed, an array containing the phone number of the account and the status of the transaction is sent through the GSM/GPRS shield. The code then sets the GSM shield to SMS mode and sends a crafted message highlighting the status of the transaction to the vehicle owner's phone. Below is a code snippet of such activity

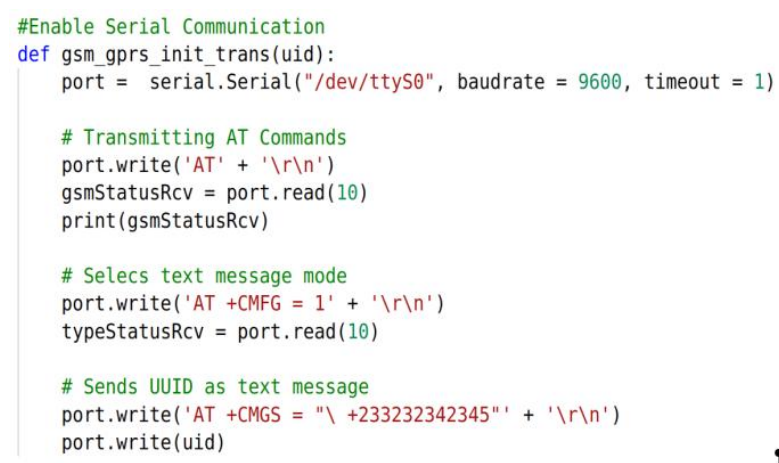

\section{Camera Image Processing}

As stated early on, there may be cases where the RFID reader is unable to read the UUID of the vehicle that uses the gate. This may be because the vehicle has not been registered with the system. In this case, the camera is activated to take an image of the vehicle after which the image is processed and sent to the database on the web server. A skeleton code is shown below.

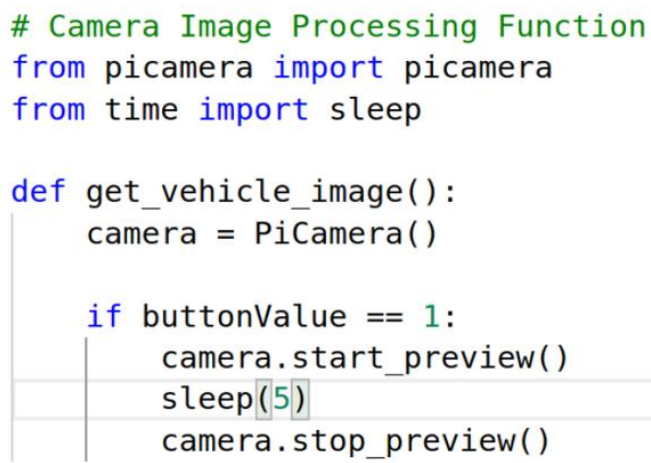

\section{RESULTS AND DISCUSSIONS}

To test the system's ability to perform as required, the following scenarios were considered:

- The first scenario, considers a registered user with enough funds in his account

- The second scenario, considers another registered user that has insufficient balance in his account.

- The third scenario, examines an unregistered user.

The GhanaToll system performance was also tested in terms of data processing speed.

A prototype environment was set up to simulate a tollgate, mainly consisting of the RFID reader connected to the microcontroller unit with the GSM shield as shown in figure 13.

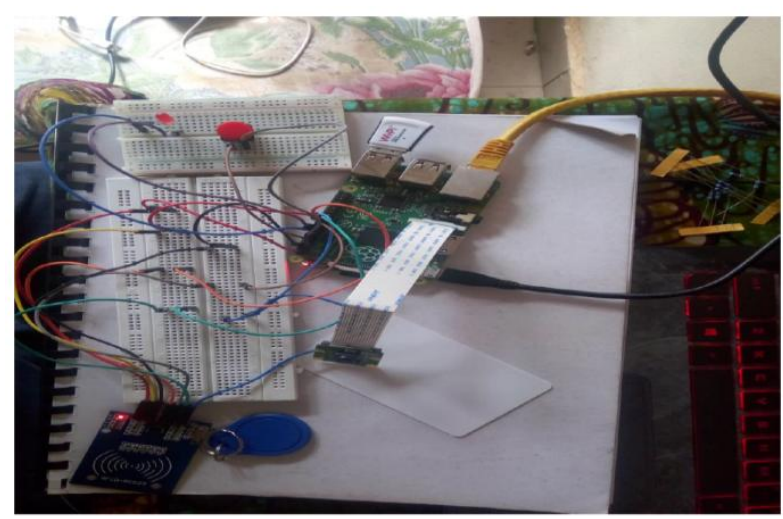

Figure 13. GhanaToll System Test Setup

In the first scenario where the user is registered with the system and has enough funds, the RFID reader reads the UUID code, which corresponds to a particular user in the system's database.

As depicted in figure 13, a simulation of the electronic toll collection system consisting of the microcontroller unit, the GSM shield, RGB LED, an LCD display and two virtual terminals were used in the setup. The microcontroller unit was mounted together with the GSM shield at the toll point or gate. It is important to note that this system of tolling does not require the vehicle to come to a complete halt as compared to other existing system.

During the time the RFID read event is taking place an interrupt allows the GSM module to send an AT command that specifies the message format to text mode and further prepares the received text to be sent to the destination specified. In this case, the destination is a mobile telephone number. The timer/ counter on the LCD display was used to estimate the time it takes for the RFID to read the vehicle's ID successfully and thereafter send relevant information to the base station after deductions are made at the backend.

After a number of tries, it was estimated that it took about 3 seconds for the reading and transmitting of the vehicle's ID read. With this, one can say that the GhanaToll electronic Toll Collection and management system met its design expectations. Data gathered from a manual tollbooth showed that it took a minimum of 2 minutes for the vehicle to get to the tollbooth. This coupled with the additional estimated minute it takes for the vendor to collect and hand out the receipt of the transaction gives a total of a minimum of 3 
minutes being spent at the toll point or gate. This compared to the GhanaToll system that estimates a 3 seconds, which even when approximated to a minute wait shows a drastic improvement in the time users spent at tollgates. The increase in the number of serviced drivers, positively affects the economy of the country, as time spent in traffic will be reduced to allow productive work to be done. Figure 14 and 15 show screenshots of various test outcomes for both registered and non-registered users

\begin{tabular}{|c|c|c|c|c|c|}
\hline \multicolumn{6}{|c|}{ Registered Toll Log } \\
\hline 10 & Regstrationtumber & Carlype & Foll & Timestamp & Location \\
\hline 5 & \& $3334 \mathrm{~m}$ & $\mathrm{~F}$ & 494 & 2017-04-17 14:50:32 & ks \\
\hline 10 & ER $464 \mathrm{~N}$ & B & 898 & 2017-04-17 21:33:12 & Newrork \\
\hline 11 & $20233 \mathrm{v}$ & D & 86 & $2017-04-19$ 11:10:35 & canada \\
\hline 262 & AS1024k & B & 598 & 2017-05-16 15:53:21 & Paris \\
\hline
\end{tabular}

Unregistered Toll Log

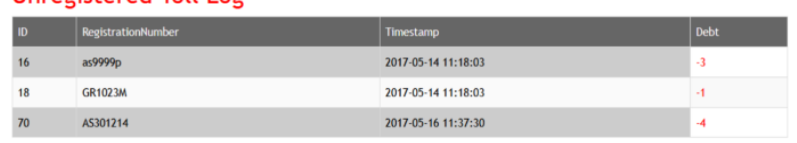

Figure 14. GhanaToll System Collection Data log

\section{Registered Owners Log}

\begin{tabular}{|c|c|c|c|c|}
\hline 10 & Nane & Adtress & Telephone & Emal \\
\hline 1 & kofi & TEMA & 501233444 & hel \\
\hline 2 & christian & koftwon & 50134788 & step.t.tvigggmail.com \\
\hline 3 & francis & takoradi & 5411234 & acquaseegrnali.com \\
\hline 4 & Degratt & obuasi & 54585859 & degegafi.o \\
\hline 5 & phinehas & kokorantumi & 93737637 & steeskdiff \\
\hline 8 & Agyepong Kvame & po knmim 7990 & 203340409 & jundmênsim.com \\
\hline 9 & President Donald Trump & Kumasi & 233501360330 & step.twigegnail.com \\
\hline
\end{tabular}

Figure 15. GhanaToll System End User Data log

\section{CONCLUSION}

This project development has unearthed a high level of unaccountability in the current Ghanaian method of toll collection. Considering the various methods of toll collection in other parts of the world this paper came up with a local solution for Ghana and possibly the whole of West Africa that ensured more credibility, i.e., a system that kept recordings of every transaction made at the toll points or gates whilst considering the cost as well.

After a detailed analysis of the various communication technologies, available, wireless communication was selected, as it did not necessitate a physical connection between the communicating nodes. Taking into consideration the fact that the server that will store the information about the various tollbooths and their transactions will be hosted on an online server, running a cable from the tollgate to the online database seemed very unreasonable. Additionally, it offered some speed and flexibility- a wired connection would not have provided. This generally reduced the time that would be spend at the tollgates. In addition, credibility is addressed because of proper records keeping. There is a high likelihood that there will be higher customer satisfaction as the system allows the end users to monitor and have control over the money spent at the toll station.

Considering the fact that faults are not always planned it is essential to have a backup plan if they do. Taking into account the fact that wireless communication is used, a proper backup will be to have a system that does not rely on WIFI so that in cases where the server may be down proper accounts can be kept. In this vein, an additional RFID card is recommended with a reader that can be kept along the sides of the road to keep a $\log$ of the vehicles that have used the tollgates. Also, a camera with a local server can be used as a backup so that an image of the license plates of the various vehicles that use the system is recorded and kept on say a local server so that when the main system resumes its functionality, the data stored on the local server can be sent to the centralized database.

\section{REFERENCES}

[1] Preprah, Jan. 30, 2018. Three GHA employees charged with stealing GHS1,288,824.00, ghananewsagency.org. Available: https://www.ghananewsagency.org/humaninterest/t hree-gha-employees-charged-with-stealing-gh1-28 8-824-00--128032.

[2] Ghanaweb.com, Sept. 1, 2019, Electronic Toll Collection Commerces on Accra-Tema Motorway. Available: https://www.ghanaweb.com/GhanaHomePage/NewsArch ive/Electronic-toll-collection-commences-on-AccraTema-Motorway-167897

[3] Hagan, S, Nov. 10, 2019. Electronic Toll Collection System: A necessity for Ghana (Part 1). Institute of ICT ProfessionalsGhana..Available:https://iipgh.org/electroni c-toll-collection-s ystem-a-necessity-for-ghana-part-1

[4] The Finderonline.com, May 10, 2017. Maximizing revenue from tollbooths; Automation the answer Available:

https://www.thefinderonline.com/feature/item/7772maximising-revenue-from-toll-booths-automation-theanswer

[5] Nandimi, S and Premkumar, S, 2007. Automatic Toll Gate System Using Advanced RFID and GSM Technology, International Journal of Advanced Research in Electrical and Electronics and Instrumentation Engineering, Vol. 3, no.11, pp 13002-13007.

[6] Lee,W., Tseng, S. and Wang, C., 2018. Design and implementation of electronic toll collection system based on vehicle positioning system techniques, Computer Communications, Vol. 31, no.12, pp.2925-2933.

[7] Vadali, R., Shinde, A., Ghuse, S., More and Tope, D., 2019. Secured Electronic toll collection using RFID and mobile application, Semanticscholar.org.

[8] Swathi A and Baba BM, 2018. RFID Based Automatic Toll Collection System Using GSM Technology. In: International Journal of Scientific Research in Science and Technology (IJSRST), Volume 4, Issue 5, 2018, Pages 532- 536.Print ISSN: 2395-6011 | Online ISSN: 2395-602X.

[9] Suganya R., Abinaya K, Preethi A., Raj PP and Swathika G., 2018. Automated Toll Plaza System Using RFID and GSM Technology. In: International Journal of Pure and Applied Mathematics, Volume 119 No. 12, 1679116801, ISSN: 1314-3395 (on-line version).

[10] Malaj SS and Kamate SS, 2017. RFID \& GSM Based Toll Tax System. In: International Journal of Scientific and Research Publications, Volume 7, Issue 1, January 2017.Pages 110-114. ISSN 2250-3153 